\title{
Progress report on solar age calibration
}

\author{
G. Houdek ${ }^{1}$ and D. O. Gough ${ }^{1,2}$ \\ ${ }^{1}$ Institute of Astronomy, Madingley Road, Cambridge CB3 0HA, UK \\ email: hg@ast.cam.ac.uk \\ ${ }^{2}$ Department of Applied Mathematics and Theoretical Physics, Wilberforce Road, \\ Cambridge CB3 0WA, UK \\ email: douglas@ast.cam.ac.uk
}

\begin{abstract}
We report on an ongoing investigation into a seismic calibration of solar models designed for estimating the main-sequence age and a measure of the chemical abundances of the Sun. Only modes of low degree are employed, so that with appropriate modification the procedure could be applied to other stars. We have found that, as has been anticipated, a separation of the contributions to the seismic frequencies arising from the relatively smooth, glitch-free, background structure of the star and from glitches produced by helium ionization and the abrupt gradient change at the base of the convection zone renders the procedure more robust than earlier calibrations that fitted only raw frequencies to glitch-free asymptotics. As in the past, we use asymptotic analysis to design seismic signatures that are, to the best of our ability, contaminated as little as possible by those uncertain properties of the star that are not directly associated with age and chemical composition. The calibration itself, however, employs only numerically computed eigenfrequencies. It is based on a linear perturbation from a reference model. Two reference models have been used, one somewhat younger, the other somewhat older than the Sun. The two calibrations, which use BiSON data, are more-or-less consistent, and yield a main-sequence age $t_{\odot}=4.68 \pm 0.02 \mathrm{~Gy}$, coupled with a formal initial heavy-element abundance $Z=0.0169 \pm 0.0005$. The error analysis has not yet been completed, so the estimated precision must be taken with a pinch of salt.
\end{abstract}

Keywords. Sun: helioseismology, Sun: abundances, stars: abundances, stars: oscillations, stars: fundamental parameters.

\section{Introduction}

The only way by which the age of the Sun can be estimated to a useful degree of precision is by accepting the basic tenets of solar-evolution theory and measuring those aspects of the structure of the Sun that are predicted by the theory to be indicators of age. The structure measurements must be carried out seismologically, and evidently one expects greatest reliability of the results when all the available helioseismic data are employed. However, the most relevant modes are those of lowest degree, because it is they that penetrate most deeply into the energy-generating core where the relic heliumabundance variation records the integrated history of nuclear transmutation. Moreover, it is also only they that can be measured in other stars. Therefore, there has been some interest in calibrating theoretical stellar models using only low-degree modes. The prospect was first discussed in detail by Christensen-Dalsgaard $(1984,88)$, Ulrich (1986) and Gough (1987), although prior to that it had already been pointed out that the helioseismic frequency data that were available at the time indicated that either the initial helium abundance $Y_{0}$ or the age $t_{\odot}$, or both, are somewhat greater than the generally accepted values (Gough 1983), an inference which is consistent with our present findings. Subsequent, more careful, calibrations were carried out by Guenther (1989), Gough \& Novotny (1990), Guenther \& Demarque (1997), Weiss \& Schlattl (1998), Dziembowski 
et al. (1999), Gough (2001) and Bonanno, Schlattl \& Paternò (2002). Not all of them addressed the influence of uncertainties in $Y_{0}$ on the determination of $t_{\odot}$.

As a main-sequence star ages, helium is produced in the core, increasing the mean molecular mass $\mu$, preferentially at the centre, and thereby reducing the sound speed. The resulting functional form of the sound speed $c(r)$ depends not only on age $t_{\odot}$ but also on the relative augmentation of $\mu(r)$, which itself depends on the initial absolute value of $\mu$, and hence on $Y_{0}$. Gough (2001) tried to separate these two effects using the degree dependence of the small separation $d_{n, l}=3(2 l+3)^{-1}\left(\nu_{n, l}-\nu_{n-1, l+2}\right)$ of cyclic frequencies $\nu_{n, l}$, where $n$ is order and $l$ is degree. This is possible, in principle, because modes of different degree and similar frequency sample the core differently. However, that difference is subtle, and the sensitivity to the relatively fine distinction between the effects of $t_{\odot}$ and $Y_{0}$ on the functional form of $c(r)$ in the core is low. Consequently the error in the calibration produced by errors in the observed frequency data is uncomfortably high.

This lack of sensitivity can be overcome by using, in addition to core-sensitive seismic signatures, the relatively small oscillatory component of the eigenfrequencies induced by the sound-speed glitch associated with helium ionization (Gough 2002), whose amplitude is close to being proportional to helium abundance $Y$ (Houdek \& Gough 2007a). The neglect of that component in the previously employed asymptotic signature $d_{n, l}$ had not only omitted an important diagnostic of $Y$ but had also imprinted an oscillatory contamination in the calibration as the limits $\left(k_{1}, k_{2}\right)$, where $k=n+\frac{1}{2} l$, of the adopted mode range was varied (Gough 2001). It therefore behoves us to decontaminate the core signature from glitch contributions produced in the outer layers of the star (from both helium ionization and the abrupt variation at the base of the convection zone, and also from hydrogen ionization and the superadiabatic convective boundary layer immediately beneath the photosphere). To this end a helioseismic glitch signature has been developed by Houdek \& Gough (2007a), from which frequency contributions $\delta \nu_{n, l}$ can be computed and subtracted from the raw frequencies $\nu_{n, l}$ to produce effective glitch-free frequencies $\nu_{\mathrm{s} n, l}$ to which a glitch-free asymptotic formula (2.10) can be fitted. The solar calibration is then accomplished by interpolating the theoretical seismic signatures computed on a grid of solar models to the observations, using a standard grid to compute derivatives with respect to $t_{\odot}$ and $Y_{0}$, and a carefully computed reference solar model designed to be close to the Sun. The result of the first preliminary calibration by this method, using BiSON data, has been reported by Houdek \& Gough (2007b). Here we enlarge on our discussion of the analysis, and we augment our results with a calibration based on a second reference solar model.

\section{The seismic diagnostic and calibration method}

Any abrupt variation in the stratification of a star (relative to the scale of the inverse radial wavenumber of a seismic mode of oscillation), which here we call an acoustic glitch, induces an oscillatory component in the spacing of the cyclic eigenfrequencies of seismic modes. Our interest is principally in the glitch caused by the depression in the first adiabatic exponent $\gamma_{1}=(\partial \ln p / \partial \ln \rho)_{s}$ (where $p, \rho$ and $s$ are pressure, density and specific entropy) caused by helium ionization, which imparts a glitch in the sound speed $c(r)$. The deviation

$$
\delta \nu_{i}:=\nu_{i}-\nu_{\mathrm{s} i}
$$

where $i:=(n, l)$, of the eigenfrequency $\nu_{i}$ from the corresponding frequency $\nu_{\mathrm{s} i}$ of a similar smoothly stratified star is the indicator of $Y$ that we use in conjunction with the indicators of core structure to determine the main-sequence age.

Approximate expressions for the frequency contributions $\delta \nu_{i}$ arising from acoustic glitches in solar-type stars were recently presented by Houdek \& Gough (2007a). Here 


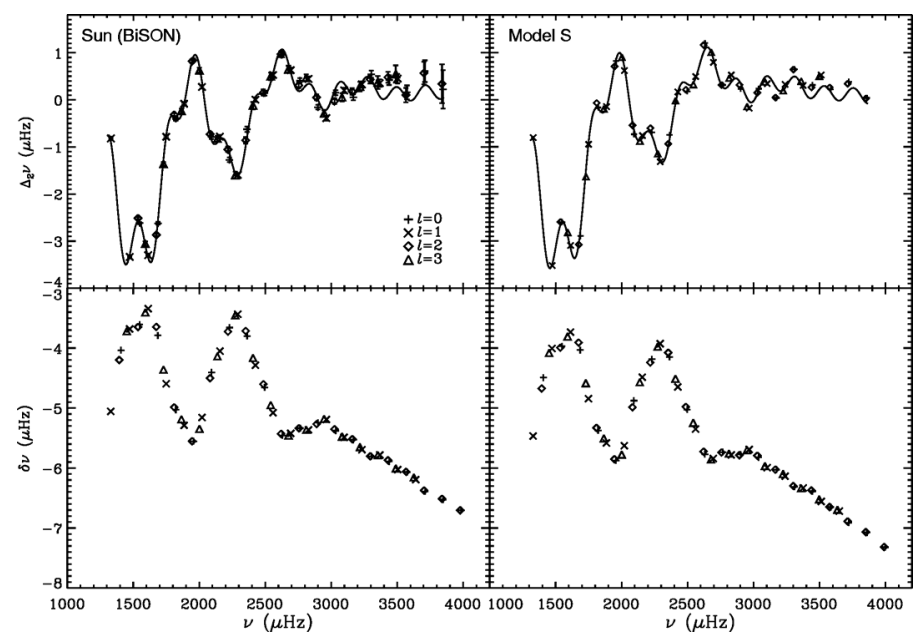

Figure 1. Top left: The symbols (with error bars obtained under the assumption that the raw frequency errors are independent) represent second differences, $\Delta_{2} \nu$, of low-degree solar frequencies from BiSON. Top right: The symbols are second differences $\Delta_{2} \nu$ of adiabatic pulsation eigenfrequencies of solar Model S of Christensen-Dalsgaard et al. (1996). The solid curves in both panels are the diagnostics $(2.2)-(2.8)$, whose eleven parameters have been adjusted to fit the data optimally. Bottom: The symbols denote contributions $\delta \nu$ to the frequencies produced by the acoustic glitches of the Sun (left panel) and Model S (right panel).

we improve them by adopting the appropriate Airy functions $\operatorname{Ai}(-x)$ that are used as comparison functions in the JWKB approximations to the oscillation eigenfunctions, as did Houdek \& Gough (2007b). The complete expression for $\delta \nu_{i}$ is then given by

$$
\delta \nu_{i}=\delta_{\gamma} \nu_{i}+\delta_{\mathrm{c}} \nu_{i}
$$

where

$$
\begin{aligned}
\delta_{\gamma} \nu= & -\sqrt{2 \pi} A_{\mathrm{II}} \Delta_{\mathrm{II}}^{-1}\left[\nu+\frac{1}{2}(m+1) \nu_{0}\right] \\
& \times\left[\tilde{\mu} \tilde{\beta} \int_{0}^{T} \kappa_{\mathrm{I}}^{-1} \mathrm{e}^{-\left(\tau-\tilde{\eta} \tau_{\mathrm{II}}\right)^{2} / 2 \tilde{\mu}^{2} \Delta_{\mathrm{II}}^{2}}|x|^{1 / 2}|\mathrm{Ai}(-x)|^{2} \mathrm{~d} \tau\right. \\
& \left.+\int_{0}^{T} \kappa_{\mathrm{II}}^{-1} \mathrm{e}^{-\left(\tau-\tau_{\mathrm{II}}\right)^{2} / 2 \Delta_{\mathrm{II}}^{2}}|x|^{1 / 2}|\mathrm{Ai}(-x)|^{2} \mathrm{~d} \tau\right]
\end{aligned}
$$

arises from the variation in $\gamma_{1}$ induced by helium ionization, and

$$
\begin{aligned}
\delta_{\mathrm{c}} \nu \simeq & A_{\mathrm{c}} \nu_{0}^{3} \nu^{-2}\left(1+1 / 16 \pi^{2} \tau_{0}^{2} \nu^{2}\right)^{-1 / 2} \\
& \times\left\{\cos \left[2 \psi_{\mathrm{c}}+\tan ^{-1}\left(4 \pi \tau_{0} \nu\right)\right]-\left(16 \pi^{2} \tilde{\tau}_{\mathrm{c}}^{2} \nu^{2}+1\right)^{1 / 2}\right\}
\end{aligned}
$$

arises from the acoustic glitch at the base of the convection zone resulting from a near discontinuity (a true discontinuity in theoretical models using local mixing-length theory with a non-zero mixing length at the lower boundary of the convection zone) in the second derivative of density. Here, $m=3.5$ is a constant, being a representative polytropic index in the expression for the approximate effective phase $\psi$ appearing in the argument of the Airy function, and $\tilde{\beta}, \tilde{\eta}$ and $\tilde{\mu}$ are constants of order unity which account for the relation between the acoustic glitches caused by the first and second stages of ionization of helium (Houdek \& Gough 2007a); $\tau$ is acoustic depth beneath the seismic surface of the star, and $T \simeq 1 / 2 \nu_{0}$ is the total acoustic radius of the star; $\Delta_{\text {II }}$ and $\tau_{\text {II }}$ are respectively the acoustic width of the glitch and its acoustic depth beneath the seismic surface. The argument of 
the Airy function is $x=\operatorname{sgn}(\psi)|3 \psi / 2|^{2 / 3}$, where

$$
\psi(\tau)=\kappa \omega \tilde{\tau}-(m+1) \cos ^{-1}[(m+1) / \omega \tilde{\tau}] \quad \text { if } \tilde{\tau}>\tau_{\mathrm{t}},
$$

and

$$
\psi(\tau)=|\kappa| \omega \tilde{\tau}-(m+1) \ln [(m+1) / \omega \tilde{\tau}+|\kappa|] \quad \text { if } \tilde{\tau} \leqslant \tau_{\mathrm{t}},
$$

in which $\tilde{\tau}=\tau+\omega^{-1} \epsilon_{\mathrm{II}}$, with $\omega=2 \pi \nu$, and $\tau_{\mathrm{t}}$ is the location of the upper turning point of the mode; also $\kappa(\tau)=\left[1-(m+1)^{2} / \omega^{2} \tilde{\tau}^{2}\right]^{1 / 2}$, and $\kappa_{\mathrm{I}}=\kappa\left(\tilde{\eta} \tau_{\mathrm{II}}\right)$ and $\kappa_{\mathrm{II}}=\kappa\left(\tau_{\mathrm{II}}\right)$. In addition

$$
\psi_{\mathrm{c}}=\kappa_{\mathrm{c}} \omega \tilde{\tau}_{\mathrm{c}}-(m+1) \cos ^{-1}\left[(m+1) / \tilde{\tau}_{\mathrm{c}} \omega\right]+\pi / 4,
$$

where $\kappa_{\mathrm{c}}=\kappa\left(\tau_{\mathrm{c}}\right)$ and $\tilde{\tau}_{\mathrm{c}}=\tau_{\mathrm{c}}+\omega^{-1} \epsilon_{\mathrm{c}}$.

The seven coefficients $\eta_{\alpha}=\left(A_{\mathrm{II}}, \Delta_{\mathrm{II}}, \tau_{\mathrm{II}}, \epsilon_{\mathrm{II}}, A_{\mathrm{c}}, \tau_{\mathrm{c}}, \epsilon_{\mathrm{c}}\right), \alpha=1, \ldots, 7$, are found by fitting the second difference

$$
\Delta_{2 i} \nu \equiv \nu_{n-1, l}-2 \nu_{n, l}+\nu_{n+1, l} \simeq \Delta_{2 i}\left(\delta_{\gamma} \nu+\delta_{\mathrm{c}} \nu\right)+\sum_{k=0}^{3} a_{k} \nu_{i}^{-k} \equiv g_{i}\left(\nu_{j} ; \eta_{\alpha}\right)
$$

to the corresponding observations by minimizing

$$
E_{\mathrm{g}}=\left(\Delta_{2 i} \nu-g_{i}\right) C_{\Delta i j}^{-1}\left(\Delta_{2 j} \nu-g_{j}\right)
$$

using the value of $\nu_{0}$ obtained by fitting to $(2.10)$, where $C_{\Delta i j}^{-1}$ is the $(i, j)$ element of the inverse of the covariance matrix $\mathrm{C}_{\Delta}$ of the observational errors in $\Delta_{2 i} \nu$, computed, perforce, under the assumption that the errors in the frequency data $\nu_{i}$ are independent. The last term in equation (2.8) approximates smooth contributions arising, in part, from wave refraction in the stellar core, from hydrogen ionization and from the superadiabaticity of the upper boundary layer of the convection zone, introducing four more fitting coefficients $a_{k}=\eta_{\alpha}, k=0, \ldots, 3, \alpha=8, \ldots, 11$. The covariance matrix $C_{\eta \alpha \gamma}$ of the errors in $\eta_{\alpha}$ were established by Monte Carlo simulation.

The outcome of the fitting to the BiSON data (Basu et al. 2007) and to the adiabatically computed eigenfrequencies of solar Model S (Christensen-Dalsgaard et al. 1996) is displayed in Figure 1: the upper panels display the second differences, together with the fitted formula (2.8), the lower panels display the corresponding contributions $\delta \nu_{i}$ to the frequencies of oscillation from the acoustic glitches. All the frequencies displayed in the figure have been used in equation (2.9) for fitting (2.8).

To the resulting glitch-free frequencies $\nu_{\mathrm{s} i}$, derived from equation (2.1), of both the solar observations and the eigenfrequencies of the reference solar model, is fitted the asymptotic expression

$\nu_{\mathrm{s} i} \sim\left(n+\frac{1}{2} l+\hat{\epsilon}\right) \nu_{0}-\frac{A L^{2}-B}{\nu_{\mathrm{s} i}} \nu_{0}^{2}-\frac{C L^{4}-D L^{2}+E}{\nu_{\mathrm{s} i}^{3}} \nu_{0}^{4}-\frac{F L^{6}-G L^{4}+H L^{2}-I}{\nu_{\mathrm{s} i}^{5}} \nu_{0}^{6} \equiv s\left(\nu_{\mathrm{s} i} ; \xi_{\beta}\right)$,

by minimizing $\left(\nu_{\mathrm{s} i}-s_{i}\right) C_{\mathrm{s} i j}^{-1}\left(\nu_{j}-s_{j}\right)$, where $L^{2}=l(l+1)$ and $\mathrm{C}_{\mathrm{s}}$ is the covariance matrix of the observational errors in $\nu_{\mathrm{s} i}$, from which we obtain both the coefficients $\xi_{\beta}=\left(\nu_{0}, \hat{\epsilon}, A, B, C, D, E, F, G, H, I\right), \beta=1, \ldots, 11$, and the covariance matrix $C_{\xi \beta \delta}$ of the errors. Following Gough (2001), we carry out this fitting in the frequency range given by $k_{1} \leqslant k \leqslant k_{2}$, where $k=n+\frac{1}{2} l$ and $0 \leqslant l \leqslant 3$, and we vary $k_{1}$ and $k_{2}$. Each of the parameters $\xi_{\beta}$ represents an integral of a function of the equilibrium stratification. The integrals $A, C$ and $F$ are of particular importance to our analysis, because $C$ and $F$ are dominated by conditions in the core, and, although the contributions to $A$ from the core and the rest of the star are roughly equal in magnitude (and potentially have opposite signs), the latter is relatively insensitive to $t_{\odot}$ and $Y_{0}$. The integrands in the remaining integrals are either more evenly distributed throughout the Sun or are concentrated near the surface. 
Table 1. Partial derivatives $H_{\alpha j}$ obtained from two sets of calibrated evolutionary models for the Sun. Values with respect to age $t_{\odot}$ are in units of $\mathrm{Gy}^{-1}$.

\begin{tabular}{cccccc}
\hline$\left(\partial A / \partial t_{\odot}\right)_{Z}$ & $(\partial A / \partial Z)_{t_{\odot}}$ & $\left(\partial C / \partial t_{\odot}\right)_{Z}$ & $(\partial C / \partial Z)_{t}$ & {$\left[\partial\left(-\delta \gamma_{1} / \gamma_{1}\right) / \partial t_{\odot}\right]_{Z}$} & {$\left[\partial\left(-\delta \gamma_{1} / \gamma_{1}\right) / \partial Z\right]_{t}$} \\
\hline-0.0469 & -0.584 & 0.677 & 36.8 & -0.00656 & 0.442 \\
\hline
\end{tabular}

We have carried out age calibrations using combinations of the parameters

$$
\zeta_{\alpha}=\left(A, C,-\delta \gamma_{1} / \gamma_{1}\right), \quad \alpha=1,2,3,
$$

where $-\delta \gamma_{1} / \gamma_{1}=A_{\mathrm{II}} / \sqrt{2 \pi} \nu_{0} \Delta_{\mathrm{II}}$ is a measure of the maximum depression in $\gamma_{1}$ in the second helium ionization zone. Presuming, as is normal, that the reference model is parametrically close to the Sun, we consider the reference value $\zeta_{\alpha}^{\mathrm{r}}$ to be approximated by a two-term Taylor expansion of $\zeta_{\alpha}$ about the value $\zeta_{\alpha}^{\odot}$ of the Sun:

$$
\zeta_{\alpha}^{\mathrm{r}}=\zeta_{\alpha}^{\odot}-\left(\frac{\partial \zeta_{\alpha}}{\partial t_{\odot}}\right)_{Z} \Delta t_{\odot}-\left(\frac{\partial \zeta_{\alpha}}{\partial Z}\right)_{t_{\odot}} \Delta Z+\epsilon_{\zeta \alpha}
$$

where $\Delta t_{\odot}$ and $\Delta Z$ are the deviations of age $t_{\odot}$ and initial heavy-element abundance $Z$ from the reference model, and $\epsilon_{\zeta \alpha}$ are the formal errors in the calibration parameters whose covariance matrix $C_{\zeta \alpha \beta}$ can be derived from $C_{\xi \beta \delta}$ and $C_{\eta \alpha \gamma}$. A (parametrically local) maximum-likelihood fit then leads to the following set of linear equations:

$$
H_{\alpha j} C_{\zeta \alpha \beta}^{-1} H_{\beta k} \Theta_{0 k}=H_{\alpha j} C_{\zeta \alpha \beta}^{-1} \Delta_{0 \beta},
$$

in which $\Theta_{k}=\left(\Delta t_{\odot}, \Delta Z\right)+\epsilon_{\Theta k}=\Theta_{0 k}+\epsilon_{\Theta k}, k=1,2$, is the solution vector subject to (correlated) errors $\epsilon_{\Theta k}, \Delta_{\beta}=\zeta_{\beta}^{\odot}-\zeta_{\beta}^{\mathrm{r}}+\epsilon_{\zeta \beta}=\Delta_{0 \beta}+\epsilon_{\zeta \beta}$, and the partial derivatives $H_{\alpha j}=\left[\left(\partial \zeta_{\alpha} / \partial t_{\odot}\right)_{Z},\left(\partial \zeta_{\alpha} / \partial Z\right)_{t_{\odot}}\right], j=1,2$.

A similar set of equations is obtained for the formal errors $\epsilon_{\Theta k}$ :

$$
H_{\alpha j} C_{\zeta \alpha \beta}^{-1} H_{\beta k} \epsilon_{\Theta k}=H_{\alpha j} C_{\zeta \alpha \beta}^{-1} \epsilon_{\zeta \beta},
$$

from which the error covariance matrix $C_{\Theta k q}=\overline{\epsilon_{\Theta k} \epsilon_{\Theta q}}$ can be computed from $C_{\zeta \alpha \beta}$.

The partial derivatives $H_{\alpha j}$ were obtained from the two sets of five calibrated evolutionary models for the Sun that were used in a similar calibration by Houdek \& Gough (2007b), computed with the evolutionary programme by Christensen-Dalsgaard (1982), and adopting the Livermoore equation of state and the OPAL92 opacities. One set of models has a constant value for the heavy-element abundance $Z=0.02$ but varying age; the other has constant age but varying $Z$. Note that, for prescribed relative abundances of heavy elements, the condition that the luminosity and radius of the Sun agree with observation defines a functional relation between $Y_{0}, Z$ and $t_{\odot}$. The values of the partial derivatives $H_{\alpha j}$ are listed in Table 1 .

\section{Results}

To illustrate the effect of taking $\delta \nu_{i}$ into account, we compare in Figure 2 a first assessment of $A\left(k_{1}, k_{2}\right)$ using the glitch-free frequencies $\nu_{\mathrm{s} i}$ (left panel) with that obtained from the raw frequencies $\nu_{i}$ (right panel). Recall that $A$ represents a functional of the equilibrium structure of the star, and should not vary with $k_{1}$ and $k_{2}$. The range of values for $A$ is the lower for $\nu_{\mathrm{s} i}$, as we had anticipated. We believe that the upturn of $A$ for low values of $k_{1}$ in the left panel of the figure is a result of the failure of the asymptotic formula (2.2)-(2.4) when $\nu_{i}$ is low. The dipping of $A$ at high values of $k_{1}$ and low values of $k_{2}$ occurs because the frequency range is too small for a reliable determination of the fitting coefficients $\xi_{\beta}$. We therefore adopt intermediate values for $k_{1}$ and high values for $k_{2}$, for which $A$ is insensitive to the selected frequency range. 

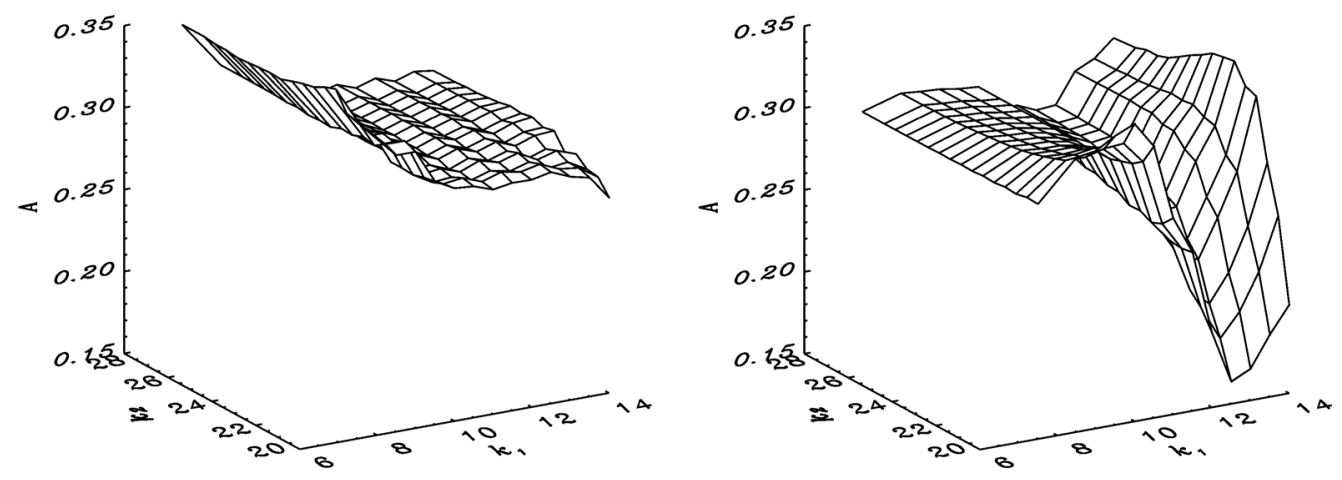

Figure 2. Asymptotic fitting coefficient $A$ (see equation 2.10) as functions of $k_{1}$ and $k_{2}$ $\left(k=n+\frac{1}{2} l\right)$. Results are shown for fitting (2.10) to the glitch-free frequencies $\nu_{\mathrm{s} i}$ (left panel) and to the raw frequencies $\nu_{i}$ (right panel).

Age calibrations using different combinations of the parameters $\zeta_{\alpha}$ and two different reference models are summarized in Table 2 . The younger reference model is 'Model S' (Christensen-Dalsgaard et al. 1996) which has age $t_{\odot}=4.6 \mathrm{~Gy}$; the second is 'Model T', which has age $t_{\odot}=4.7 \mathrm{~Gy}$. The same physics was adopted in the evolutionary calculations of both models. We notice in Table 2 that the calibration for the combination $(A, C)$, i.e. without $\delta \gamma_{1} / \gamma_{1}$, is less stable to a change in the reference model than are the calibrations with combinations in which $\delta \gamma_{1} / \gamma_{1}$ is included, and therefore is less reliable, as we have explained in the introduction. If we ignore in Table 2 the results for $(A, C)$ and combine the others, we obtain

$$
t_{\odot}=4.68 \pm 0.02 \mathrm{~Gy}, \quad Z=0.0169 \pm 0.0005 .
$$

Including the calibrations with $(A, C)$ does not change the outcome. Error contours corresponding to the calibration from Model $S$ in the first row of Table 2 are plotted in Figure 3. Corresponding contours for Model T are the same, except that their centres are displaced to (4.677 Gy, 0.0170). One can adduce from our description of the analysis in Section 2 that our current treatment of the errors is not completely unbiassed; however, the potential bias is of the order of only $\left|\delta \nu_{i} / \nu_{i}\right|$, which is small.

The age we have found is greater than currently accepted values. The values of $Z$ are somewhat smaller than those of Models S and T (0.01963), but we hasten to point out that they should not be regarded strictly as statements about the initial heavy-element abundance, but rather as measures of the opacity in the radiative interior. Asplund et al. (2004) have argued that the photospheric abundances of C, N and O had previously been overestimated, suggesting that the actual total heavy-element abundance is even lower than had previously been believed. However, that cannot imply that the opacity in the solar interior is necessarily comparably lower because it has been implicitly calibrated here (by accepting the tenets of solar-evolution theory, and the OPAL opacity calculations upon which the models are based), and indeed the opacity has already been determined seismologically from a broader spectrum of modes than has been adopted here (Gough 2004). The matter raised by Asplund et al. therefore challenges either the opacity calculations, the nuclear reaction rates, or the basic physics of stellar evolution, not helioseismology as some spectators have surmised. As we know already from seismological structure inversions, the solar models are not accurate by helioseismological standards. Therefore the properties inferred from these calibrations could be more contaminated by systematic error than by errors in the observed frequencies. 
Table 2. Age calibrations with different combinations of $\zeta_{\alpha}$ and for the two reference models: Model $\mathrm{S}$ with an age $t_{\odot}=4.6 \mathrm{~Gy}$ and Model $\mathrm{T}$ with an age $t_{\odot}=4.7 \mathrm{~Gy}$. The first two columns show the results adopting Model S as the reference model, the third and fourth columns display the results for Model T.

\begin{tabular}{lccccccc}
\hline \multicolumn{1}{c}{$\zeta_{\alpha}$} & $t_{\odot}(\mathrm{Gy})$ & $Z$ & $t_{\odot}(\mathrm{Gy})$ & $Z$ & $C_{\Theta 11}^{1 / 2}$ & $-\left(-C_{\Theta 12}\right)^{1 / 2}$ & $C_{\Theta 22}^{1 / 2}$ \\
\hline$A, C,-\delta \gamma_{1} / \gamma_{1}$ & 4.679 & 0.0169 & 4.677 & 0.0170 & 0.017 & -0.0023 & 0.0005 \\
$A, C$ & 4.658 & 0.0177 & 4.673 & 0.0171 & 0.023 & -0.0037 & 0.0007 \\
$A,-\delta \gamma_{1} / \gamma_{1}$ & 4.673 & 0.0165 & 4.676 & 0.0169 & 0.017 & -0.0019 & 0.0007 \\
$C,-\delta \gamma_{1} / \gamma_{1}$ & 4.700 & 0.0169 & 4.680 & 0.0170 & 0.028 & -0.0029 & 0.0005 \\
\hline
\end{tabular}

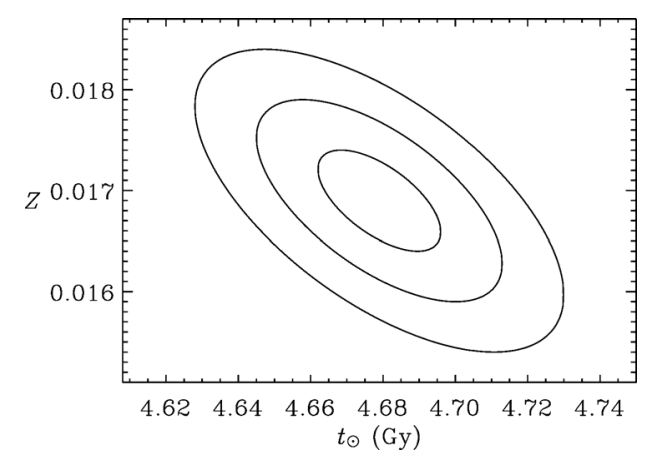

Figure 3. Error ellipses for the calibration using all three parameters $\zeta_{\alpha}$ and Models $\mathrm{S}$ as the reference model: solutions $\left(t_{\odot}, Z\right)$ satisfying the frequency data within 1,2 and 3 standard errors in those data reside in the inner, intermediate and outer ellipses, respectively.

\section{Acknowledgements}

We thank Jørgen Christensen-Dalsgaard for providing us with his stellar-evolutionary programme. GH acknowledges support by the STFC of the UK.

\section{References}

Asplund, M., Grevesse, N., Sauval, A. J., Allende Prieto, C., \& Kiselman, D. 2004, A\&A 417, 751

Basu, S., Chaplin, W. J., Elsworth, Y., New, A. M., Serenelli, G., \& Verner, G. A. 2007, ApJ 655,660

Bonanno, A., Schlattl, H., \& Paternò, L. 2002, A\&A A 390, 1115

Christensen-Dalsgaard, J. 1982, MNRAS 199, 735

Christensen-Dalsgaard, J. 1984, in: Mangeney, A., Praderie, F., (eds), Space Research Prospects in Stellar Activity and Variability, Paris Observatory Press, Paris, p. 11

Christensen-Dalsgaard, J. 1988, in: Christensen-Dalsgaard, J., Frandsen, S., (eds), Proc. IAU Symp. 123, Advances in helio- and asteroseismology, Reidel, Dordrecht, p. 295

Christensen-Dalsgaard, J. et al. 1996, Sci 272, 1286

Dziembowski, W. A., Fiorentini, G., Ricci, B., \& Sienkiewicz, R. 1999, A\&GA 343, 990

Gough, D. O. 1983, in: Shaver, P. A., Kunth, D., Kjär, K., (eds), Primordial helium, Southern Observatory, p. 117

Gough, D. O. 1987, Nat. 326, 257

Gough, D. O. 2001, in: von Hippel, T., Simpson, C., Manset, N., (eds), ASP Conf. Ser. Vol. 245, Astrophysical ages and timescales, Gough D. O. 1987, Nat. 326, 257

Gough, D. O. 2002, in: Favata, F., Roxburgh, I. W., Gadalí-Enríquez, D., (eds), Proc 1st Eddington Workshop: Stellar structure and habitable planet finding, ESA SP-485, Noordwijk, p. 65

Gough, D. O. 2004, in: Čelebonović, V., Däppen, W., Gough, D. O., (eds), AIP Conf. Proc. Vol. 731, Equation-of-state and phase-transition issues in models of ordinary astrophysical matter, Am. Inst. Phys., Melville, p. 119

Gough, D. O. \& Novotny, E. 1990, Solar Phys., 128, 143

Guenther, D. B. 1989, ApJ 339, 1156 
Guenther, D. B. \& Demarque, P. 1997, ApJ 484, 937

Houdek, G. \& Gough, D. O. 2007a, MNRAS 375, 861

Houdek, G. \& Gough, D. O. 2007b, in: Stancliffe, R. J., Dewi, J., Houdek, G., Martin, R. G., Tout, C. A., (eds), AIP Conf. Proc.: Unsolved Problems in Stellar Physics, American Institute of Physics, New York, p. 219

Ulrich, R. K. 1986, ApJ 306, L37

Weiss, A. \& Schlattl, H. 1998, A\&\& A32, 215

\section{Discussion}

Christensen-DalsgaArd: A comment: with SONG we expect to be able to carry out a similar analysis of distant stars, on which we of course know much less a priori.

HoudeK: This seismic diagnostic has been developed with the aim to be able to use it also for distant stars. The accuracy of the observed frequency data required for such a diagnostic analysis is one part in $10^{4}$ or better.

S. VAUCLAIR: Two small comments which are actually more relevant for stars that are slightly more massive than the Sun; First, I would like to point out that in case of helium settling below the convective zone the effect of the helium gradient in the second differences may become more important than the convective border, and than the effect of helium ionization. Second, the so-called asymptotic theory, which is very useful, may become quite wrong in some cases, at the end of the main sequence or the beginning of the sub-giant branch. The small frequency separation, which is always positive in the asymptotic theory, can become negative.

Gough \& Houdek: You are certainly correct in implying that the amplitude of the oscillatory contribution to the second differences arising from helium settling beneath the convection zone can be greater in stars more massive than the Sun, which have shallower convection zones, although whether or not it is more important than the ionization signature depends upon the issue in question. The cumulative amount of settling increases with time, and therefore is a potential indicator of age. But the sound-speed profile that it produces depends on uncertain fluid-dynamical issues associated with the tachocline, the recession of the convection zone, and possible overshooting, so we would be wary of attempting to use its seismic signature in an age calibration. In the current state of our understanding we would instead prefer to separate it from the ionization signature and then ignore it, as we have for the Sun; that course is possible provided that the helium ionization zone is acoustically far from the base of the convection zone. We would use it separately to investigate tachocline structure, however, as indeed we are in the process of doing for the Sun.

One cannot deny that conditions in some stars might be such as to render it impossible to develop an adequate asymptotic theory of low-degree acoustic modes, although we do not share your apparently implied pessimism. It is perhaps worth pointing out that there have been instances when an asymptotic formula developed in one set of circumstances has been misused by applying it without modification in another, in which the conditions for the validity of the theory are not satisfied; if that is what you mean by "so-called asymptotic theory" we must surely agree. We must point out, however, that it is not true that even the simple asymptotic glitch-free formula (2.10) precludes a negative so-called small frequency separation. 